\title{
Glicosaminoglicanos Sulfatados no Tecido Peri-uretral de Mulheres com e sem Incontinência Urinária de Esforço, no Menacme ena Pós-menopausa
}

Autor: Paulo Cezar Feldner Jr

Orientador: Prof. Dr. Manoel João Batista Castello Girão

Tese apresentada à Universidade Federal de São Paulo - Escola Paulista de Medicina para a obtenção do título de Mestre em Ginecologia em 28 de novembro de 2002.

Objetivos: caracterizar e quantificar os subtipos de glicosaminoglicanos sulfatados (GAGs) existentes no tecido peri-uretral de pacientes com e sem incontinência urinária de esforço (IUE), no menacme e na pósmenopausa.

Metodologia: Foram incluídas 40 pacientes que se submeteram a cirurgia vaginal para correção da incontinência urinária de esforço e/ou distopias genitais no período de setembro de 2001 a julho de 2002 . As pacientes foram avaliadas por anamnese padronizada, exame físico e urodinâmico e agrupadas segundo a existência de IUE e estado hormonal. Durante o procedimento cirúrgico, amostras de aproximadamente $1,0 \mathrm{x}$ $1,0 \mathrm{~cm}$ do tecido peri-uretral foram retiradas para avaliação. Os GAGs foram extraídos do tecido por proteólise e precipitação por ácido tricloroacético e caracterizados por eletroforese em gel de agarose. A quantificação foi feita por meio de densitometria a 525 nm do gel corado com azul de toluidina. Compararam- se os dados pela análise de variância (ANOVA), teste de Tukey e teste de $\mathrm{t}$ pareado.

Resultados: em todos os grupos estudados, houve maior predomínio de dermatam sulfato (DS), em torno de $85 \%$ do total de GAGs, seguido do condroitim sulfato (CS) e do heparam sulfato (HS). Observou-se diminuição significativa dos GAGs totais e do DS em mulheres continentes na pós-menopausa em relação às continentes no menacme. Identificou-se aumento significativo do conteúdo de GAGs total e de DS em mulheres incontinentes na pós-menopausa. Não se observou diferença significante com relação ao HS.

Conclusões: neste estudo demonstraram-se diferenças na matriz extracelular do tecido peri-uretral com aumento de GAGs totais e DS nas mulheres com incontinência urinária de esforço.

Palavras-chave: Incontinência urinária de esforço. Matriz extra-celular. Glicosaminoglicanos. Menopausa. 